Proceedings of the Edinburgh Mathematical Society (2007) 50, 277-292 (C)

DOI:10.1017/S0013091505000933 Printed in the United Kingdom

\title{
TRANSPLANTATION FORMULAE AND HADAMARD'S METHOD OF DESCENT
}

\author{
WILLIAM O. BRAY \\ Department of Mathematics $\mathcal{G}$ Statistics, University of Maine, \\ Orono, ME 04469-5752, USA (bray@math.umaine.edu)
}

(Received 23 June 2005)

\begin{abstract}
We define a partial Radon transform mapping functions on $\mathbb{R}^{n+l}$ to functions on $\mathbb{R}^{n}$ which intertwines the Laplace operator on the two spaces. As a consequence, transplantation formulae relating the radial eigenfunctions of the Laplacian on Euclidean spaces of different dimensions are obtained. Our formulae provide a geometric interpretation of integral formulae for Bessel functions of Abel type, which are found useful in potential theory. The formulae portray a view of Hadamard's method of descent within the realm of harmonic analysis, allowing the transplant of local problems from even dimensions to odd dimensions and unifying the techniques of several authors.
\end{abstract}

Keywords: Fourier transform; Bessel functions; method of descent

2000 Mathematics subject classification: Primary 42B10

\section{Introduction}

Local problems in analysis on Euclidean space often possess simpler solutions when the underlying dimension is odd. The analytic structural reason for this is that the spherical function takes an elementary form allowing reduction to a one-dimensional problem. A well-known illustration of these statements lies in the solution to the Cauchy problem for the wave equation on $\mathbb{R}^{n}$. Let $u=u(x, t)$. The initial-value problem of interest is

$$
\begin{aligned}
& \partial_{t}^{2} u=\Delta u, \quad x \in \mathbb{R}^{n}, t>0, \\
& \text { IC }:\left\{\begin{array}{c}
u(x, 0)=0, \\
u_{t}(x, 0)=f(x),
\end{array}\right\}
\end{aligned}
$$

where $\partial_{t}=\partial / \partial t, \Delta$ is the Laplace operator, and $f$ is a suitable function defined on $\mathbb{R}^{n}$, e.g. smooth with compact support. For simplicity of illustration, set $n=3$. The spherical means of a function $g$ are defined by

$$
\bar{g}_{(x)}(r)=\frac{1}{4 \pi} \int_{S^{2}} g(x+r \omega) \mathrm{d} \omega,
$$


and an application of the divergence theorem yields the intertwining property

$$
\overline{(\Delta g)}_{(x)}(r)=\left(\partial_{r}^{2}+\frac{2}{r} \partial_{r}\right) \bar{g}_{(x)}(r) .
$$

The simple identity

$$
\partial_{r}^{2}[r h(r)]=\left(r^{-1} \partial_{r}\right)\left[r^{2} h^{\prime}(r)\right],
$$

where $h$ is a function of a real variable, then implies that $u(x, t)$ solves (1.1) if and only if the function

$$
v(r, t)=r \bar{u}_{(x)}(r, t)
$$

is the solution of the one-dimensional Cauchy problem:

$$
\begin{gathered}
\partial_{t}^{2} v=\partial_{r}^{2} v, \quad r, t>0, \\
\mathrm{BC}: v(0, t)=0, \\
\mathrm{IC}:\left\{\begin{array}{c}
v(r, 0)=0, \\
\partial_{r} v(r, 0)=r \bar{f}_{(x)}(r) .
\end{array}\right\}
\end{gathered}
$$

The latter may be solved using d'Alembert's formula and $u$ may be recovered from $v$ to provide the solution to (1.1) as

$$
u(x, t)=\partial_{r} v(0, t)=t \bar{f}_{(x)}(t) .
$$

Higher dimensions can be treated similarly (see $[\mathbf{3}]$ for details).

An alternative perspective of this reduction is available using Fourier transforms, as follows. The Fourier transform is defined by

$$
\mathcal{F}_{n} f(\xi)=\int_{\mathbb{R}^{n}} f(x) \mathrm{e}^{-\mathrm{i}(x \cdot \xi)} \mathrm{d} x .
$$

Throughout this paper, we view the Fourier transform in terms of polar variables: $\mathcal{F}_{n} f(\xi)=\mathcal{F}_{n} f(\lambda, \omega)$, where $\lambda \geqslant 0$ and $\omega \in S^{n-1}$. In the case of radial functions, the Fourier transform is written $\mathcal{F}_{n} f(\lambda)$. Again taking $n=3$, we write the Fourier inversion formula in the form

$$
f(x)=\frac{1}{4 \pi^{2}} \int_{0}^{\infty}\left(f \star \phi_{\lambda}\right)(x) \lambda^{2} \mathrm{~d} \lambda,
$$

where ' $*$ ' denotes convolution and $\phi_{\lambda}(x)$ is the spherical function on $\mathbb{R}^{3}$. The latter is the unique radial solution of the equation $\Delta u+\lambda^{2} u=0$ which is 1 at the origin, explicitly in three dimensions,

$$
\phi_{\lambda}(x)=\frac{\sin \lambda r}{\lambda r},
$$

where $r=|x|$. In order to obtain the desired solution formula for (1.1) we need the identity $[\mathbf{1}]$

$$
\left(f \star \phi_{\lambda}\right)(x)=\mathcal{F}_{3}\left[\bar{f}_{(x)}\right](\lambda),
$$


where the Fourier transform above is of the radial function $y \rightarrow \bar{f}_{(x)}(y)$. In this vein the Fourier integral form of the solution of the Cauchy problem (1.1) is

$$
u(x, t)=\frac{1}{4 \pi^{2}} \int_{0}^{\infty} \mathcal{F}_{3}\left[\bar{f}_{(x)}\right](\lambda) \sin \lambda t \lambda \mathrm{d} \lambda .
$$

Note that

$$
\phi_{\lambda}(x)=-\frac{1}{\lambda^{2} r} \partial_{r}(\cos \lambda r) .
$$

Hence, a three-dimensional Fourier transform of a radial function $g$ can then be computed using integration by parts to obtain

$$
\mathcal{F}_{3} g(\lambda)=\frac{2 \pi}{\lambda^{2}} \mathcal{F}_{1} h(\lambda)
$$

where $h(r)=\partial_{r}(r \tilde{g}(r))$ and $\tilde{g}(r)=g(x)$. Using this in (1.8) and applying one-dimensional Fourier inversion yields the alternative derivation of (1.5).

We will now establish notation used throughout the rest of the paper and outline the above Fourier transform computation on $\mathbb{R}^{2 m+1}$. Given a radial function $g$, its realization as an even function on $\mathbb{R}$ is denoted by $\tilde{g}(r)=g(x)$, where $r=|x|$. Let $\mathrm{O}$ denote the origin in $\mathbb{R}^{n}$. The spherical function on $\mathbb{R}^{n}$ is the unique radial solution to the equation $\Delta u+\lambda^{2} u=0$, which takes value 1 at the origin. The spherical function is denoted $\phi_{\lambda}^{n}(x)$. In terms of Bessel functions this is

$$
\tilde{\phi}_{\lambda}^{n}(r)=2^{\sigma-1 / 2} \Gamma\left(\sigma+\frac{1}{2}\right)(\lambda r)^{-(\sigma-1 / 2)} J_{\sigma-1 / 2}(\lambda r),
$$

where $J_{\mu}$ is a Bessel function of the first kind, of order $\mu$, and $\sigma=\frac{1}{2}(n-1)$. The Fourier inversion formula can be expressed as $[\mathbf{1}]$

$$
f(x)=\frac{\omega_{n-1}}{(2 \pi)^{n}} \int_{0}^{\infty}\left(f \star \phi_{\lambda}^{n}\right)(x) \lambda^{n-1} \mathrm{~d} \lambda
$$

and, in analogy with (1.7), we have

$$
\left(f \star \phi_{\lambda}^{n}\right)(x)=\mathcal{F}_{n}\left[\bar{f}_{(x)}\right](\lambda) .
$$

The solution of (1.1) for $n=2 m+1$ as a Fourier integral is

$$
u(x, t)=\frac{\omega_{2 m}}{(2 \pi)^{2 m+1}} \int_{0}^{\infty} \mathcal{F}_{2 m+1}\left[\bar{f}_{(x)}\right](\lambda) \sin \lambda t \lambda^{2 m-1} \mathrm{~d} \lambda .
$$

Applying the differentiation formula for Bessel functions [7, p. 103] to the spherical function gives a stunning formula representing a shift of two dimensions via a differential operator:

$$
\left(r^{-1} \partial_{r}\right) \tilde{\phi}_{\lambda}^{n-2}(r)=-\frac{\lambda^{2}}{n} \tilde{\phi}_{\lambda}^{n}(r) .
$$

If $n=2 m+1$ is odd, the formula iterates to

$$
\lambda^{2 m} \tilde{\phi}_{\lambda}^{2 m+1}(r)=\frac{(2 m+1) !}{2^{m} m !}\left(-r^{-1} \partial_{r}\right)^{m} \cos \lambda r
$$


This is a variant of a well-known formula for Bessel functions of half-integer order [7, p. 111]. Substituting this formula into (1.11) (in the computation of the Fourier transform), integrating by parts and collecting constants reduces the integral to a onedimensional Fourier inversion and yields

$$
u(x, t)=\frac{2^{m-1}(m-1) !}{(2 m-1) !}\left(t^{-1} \partial_{t}\right)^{m-1}\left[t^{2 m-1} \bar{f}_{(x)}(t)\right] .
$$

This is the well-known Kirchhoff-Poisson formula; a Fourier analytic derivation in even dimensions is given at the beginning of $\S 3$.

The analysis in the preceding paragraphs depicts the idea of reducing problems to one dimension, a technique which has been applied in a variety of problems and settings: pointwise inversion of Fourier transforms [8], Paley-Wiener theorems [11] and the waveequation approach to Fourier inversion [9]. In its direct form, the technique works well in odd dimensions; even dimensions require further analysis $[\mathbf{1}, \mathbf{2}, \mathbf{8}, \mathbf{9}]$. In the case of the Cauchy problem for the wave equation (1.1) in even dimensions, this analysis is usually carried out applying the method of descent: a principle named and used extensively by Hadamard [4]. One perspective of the current paper is to provide a view of Hadamard's method in the context of harmonic analysis. We define a partial Radon transform mapping functions on $\mathbb{R}^{n+l}$ to functions on $\mathbb{R}^{n}$ which intertwines the respective Laplace operators. Applied to radial functions (see $\S 2$ ), this provides formulae generalizing the reduction formula (1.12); we call such formulae transplantation formulae. When $n$ is even, the formulae give a direct method to lift local problems of harmonic analysis in even dimensions to odd dimensions (see $\S 3$ ). Expressed in terms of Bessel functions, our transplantation formulae (see (2.9)) provide a geometric interpretation of Abel-type integral formulae for Bessel functions [12, p. 45]. A natural dual transform is constructed along with associated dual transplantation formulae. These provide a geometric interpretation of a formula for Bessel functions that is important in the study of Bochner-Riesz summability $[\mathbf{1 0}]$ and in potential theory. In $\S 3$ we apply the transplantation formulae to provide an alternative approach to, and extension of, results of Pinsky and Taylor [9] on Fourier inversion at a point, and an alternative proof of Helgason's [5] Paley-Wiener theorem.

The idea for this paper is based on [2], in which transplantation formulae were obtained analytically in the setting of symmetric spaces. Geometric perspectives on the latter will appear elsewhere.

\section{Transplantation formulae}

Let $x \in \mathbb{R}^{n+l}$, where $l \geqslant 1$, and write $x=\left(x^{\prime}, x^{\prime \prime}\right)$, where $x^{\prime} \in \mathbb{R}^{n}$ and $x^{\prime \prime} \in \mathbb{R}^{l}$. For measurable functions $f$ defined on $\mathbb{R}^{n+l}$, define

$$
T_{l} f\left(x^{\prime}\right)=T_{l}\left(x^{\prime}, x^{\prime \prime}\right)=\int_{\mathbb{R}^{l}} f\left(x^{\prime}, x^{\prime \prime}+\tau\right) \mathrm{d} \tau .
$$

The following key properties are readily apparent from our definition. 
(i) If $f$ is $\mathrm{SO}(n+l)$-invariant (radial), then $T_{l} f$ is $\mathrm{SO}(n)$-invariant.

(ii) Let $\Delta_{n}$ denote the Laplacian on $\mathbb{R}^{n}$. Then $T_{l}$ intertwines the Laplacian on $\mathbb{R}^{n+l}$ and $\mathbb{R}^{n}$, i.e.

$$
\Delta_{n} T_{l} f=T_{l} \Delta_{n+l} f
$$

Using the notation of $\S 1$, these properties suggest a transplantation formula of the form

$$
\phi_{\lambda}^{n}=d(\lambda, l, n) T_{l} \phi_{\lambda}^{n+l}
$$

for some function $d(\lambda, l, n)$ independent of $x^{\prime}$. Before giving this result, we define a dual transform as follows. Given a function $g$ on $\mathbb{R}^{n}$, its Hadamard extension is defined by $H g\left(x^{\prime}, x^{\prime \prime}\right)=g\left(x^{\prime}\right)$. It is immediate that

$$
\int_{\mathbb{R}^{n+l}} f(x) H g(x) \mathrm{d} x=\int_{\mathbb{R}^{n}} T_{l} f\left(x^{\prime}\right) g\left(x^{\prime}\right) \mathrm{d} x^{\prime},
$$

a formula valid for all measurable functions $f$ and $g$ when either side is finite, with the functions replaced by their absolute values. Restricting to radial functions, this formula yields the desired duality:

$$
\int_{\mathbb{R}^{n+l}} f(x) T_{l}^{*} g(x) \mathrm{d} x=\int_{\mathbb{R}^{n}} T_{l} f\left(x^{\prime}\right) g\left(x^{\prime}\right) \mathrm{d} x^{\prime}
$$

where

$$
T_{l}^{*} g(x)=\frac{1}{\omega_{n+l-1}} \int_{S^{n+l-1}} H g(|x| \omega) \mathrm{d} \omega .
$$

The geometric interpretation of $T_{l}^{*} g$ is clear: it is the symmetrization about the origin of the Hadamard extension of $g$. It follows that

$$
\Delta_{n+l} T^{*} g=T_{l}^{*} \Delta_{n} g
$$

and hence we should have a formula dual to (2.3):

$$
T_{l}^{*} \phi_{\lambda}^{n}=d^{\prime}(\lambda, l, n) \phi_{\lambda}^{n+l},
$$

for a suitable function $d^{\prime}$.

Remark 2.1. Notice that the operator $T_{l}$ and its dual $T_{l}^{*}$ also depend on $n$. We have chosen not to indicate this directly in the operator symbol for clarity as the underlying dimension should be clear from the context or by hypothesis.

We can now state and prove the main results of this section.

Theorem 2.2. Let $n \geqslant 3$ and $1 \leqslant l<n-1$. Then, for $\lambda>0$, we have

$$
\phi_{\lambda}^{n}\left(x^{\prime}\right)=\frac{\Gamma(n / 2)}{2^{l} \pi^{l / 2} \Gamma((n+l) / 2)} \lambda^{l}\left(T_{l} \phi_{\lambda}^{n+l}\right)\left(x^{\prime}\right) .
$$


Proof. The conditions on $l$ and $n$ guarantee the existence of $T_{l} \phi_{\lambda}^{n+l}$ as an absolutely convergent integral and define a radial continuous function. We show that $T_{l} \phi_{\lambda}^{n+l}$ is a weak solution of $\Delta_{n} u+\lambda^{2} u=0$. Let $g \in C_{\mathrm{c}}^{\infty}\left(\mathbb{R}^{n}\right)$. Then

$$
\begin{aligned}
\int_{\mathbb{R}^{n}} \Delta_{n}\left(T_{l} \phi_{\lambda}^{n+l}\right)\left(x^{\prime}\right) g\left(x^{\prime}\right) \mathrm{d} x^{\prime} & =\int_{\mathbb{R}^{n}}\left(T_{l} \phi_{\lambda}^{n+l}\right)\left(x^{\prime}\right) \Delta_{n} g\left(x^{\prime}\right) \mathrm{d} x^{\prime} \\
& =\int_{\mathbb{R}^{n+l}} \phi_{\lambda}^{n+l}(x)\left(T_{l}^{*} \Delta_{n} g\right)(x) \mathrm{d} x \\
& =\int_{\mathbb{R}^{n+l}} \phi_{\lambda}^{n+l}(x)\left(\Delta_{n+l} T_{l}^{*} g\right)(x) \mathrm{d} x \\
& =-\lambda^{2} \int_{\mathbb{R}^{n+l}} \phi_{\lambda}^{n+l}(x)\left(T_{l}^{*} g\right)(x) \mathrm{d} x \\
& =-\lambda^{2} \int_{\mathbb{R}^{n}}\left(T_{l} \phi_{\lambda}^{n+l}\right)\left(x^{\prime}\right) g\left(x^{\prime}\right) \mathrm{d} x^{\prime} .
\end{aligned}
$$

It follows that $\Delta_{n}^{k}\left[T_{l} \phi_{\lambda}^{n+l}\right] \in C\left(\mathbb{R}^{n}\right)$ for every $k=0,1, \ldots$, and hence $T_{l} \phi_{\lambda}^{n+l}$ is smooth $[6]$. This verifies (2.3). In order to calculate $d$, we express the integral defining $T_{l}$ in polar form as

$$
T_{l} f\left(x^{\prime}\right)=\omega_{l-1} \int_{\left|x^{\prime}\right|}^{\infty} \tilde{f}(s)\left(s^{2}-\left|x^{\prime}\right|^{2}\right)^{l / 2-1} s \mathrm{~d} s .
$$

Note that the integral is absolutely convergent if, say, $\tilde{f}(s)=O\left(s^{-p}\right)$, where $p>l$. Applying this to (2.3) and setting $x^{\prime}=O$ one obtains

$$
\begin{aligned}
1 & =\omega_{l-1} d(\lambda, l, n) 2^{(n+l-2) / 2} \Gamma\left(\frac{n+l}{2}\right) \int_{0}^{\infty}(\lambda s)^{-(n+l-2) / 2} J_{(n+l-2) / 2}(\lambda s) s^{l-1} \mathrm{~d} s \\
& =\omega_{l-1} d(\lambda, l, n) 2^{(n+l-2) / 2} \Gamma\left(\frac{n+l}{2}\right) \lambda^{-l} \int_{0}^{\infty} J_{(n+l-2) / 2}(s) s^{-(n-l) / 2} \mathrm{~d} s .
\end{aligned}
$$

The value of the latter integral is known (see, $[\mathbf{7}$, p. 142]) and is given by

$$
\int_{0}^{\infty} J_{(n+l-2) / 2}(s) s^{-(n-l) / 2} \mathrm{~d} s=\frac{\Gamma\left(\frac{1}{2} l\right)}{2^{(n-l) / 2} \Gamma\left(\frac{1}{2} n\right)} .
$$

Substituting this and the formula $\omega_{l-1}=2 \pi^{l / 2} / \Gamma\left(\frac{1}{2} l\right)$ into the previous equation and simplifying gives

$$
d(\lambda, l, n)=\frac{\Gamma\left(\frac{1}{2} n\right)}{2^{l} \pi^{l / 2} \Gamma\left(\frac{1}{2}(n+l)\right)} \lambda^{l},
$$

as desired.

Two special cases of the above result are worth recording in explicit form corresponding to $l=1$ and $l=2$. 
Corollary 2.3. Let $\lambda>0$. Then, for $n \geqslant 3$,

$$
\begin{aligned}
& \tilde{\phi}_{\lambda}^{n}(r)=\frac{\Gamma\left(\frac{1}{2} n\right)}{\sqrt{\pi} \Gamma\left(\frac{1}{2}(n+1)\right)} \lambda \int_{r}^{\infty} \tilde{\phi}_{\lambda}^{n+1}(s)\left(s^{2}-r^{2}\right)^{-1 / 2} s \mathrm{~d} s, \\
& \tilde{\phi}_{\lambda}^{n}(r)=\frac{\lambda^{2}}{n} \int_{r}^{\infty} \tilde{\phi}_{\lambda}^{n+2}(s) s \mathrm{~d} s .
\end{aligned}
$$

Remark 2.4. Notice that (2.9) holds in the case where $n=2$; the integral needs to be interpreted in a Cauchy principal-value sense. Equation (2.10) is equivalent to the differentiation formula (1.12).

The dual to Theorem 2.2 is the following.

Theorem 2.5. Let $n \geqslant 1$ and let $l \geqslant 1$. Then

$$
T_{l}^{*} \phi_{\lambda}^{n}=\phi_{\lambda}^{n+l}
$$

Proof. In a fashion similar to that above, $T_{l}^{*} \phi_{\lambda}^{n}$ is a weak solution of $\Delta_{n+l} u+\lambda^{2} u=0$. By the same argument used in the proof of Theorem 2.2, it follows that (2.6) holds. From (2.4), or directly from (2.5),

$$
\left(\tilde{T}_{l}^{*} g\right)(s)=\frac{2}{B\left(\frac{1}{2} l, \frac{1}{2} n\right) s^{n+l-2}} \int_{0}^{s} \tilde{g}(r)\left(s^{2}-r^{2}\right)^{(l-2) / 2} r^{n-1} \mathrm{~d} r .
$$

Substituting $g=\phi_{\lambda}^{n}$, using (2.6) and setting $\lambda=0$ yields

$$
d^{\prime}(\lambda, l, n)=\frac{2}{B\left(\frac{1}{2} l, \frac{1}{2} n\right) s^{n+l-2}} \int_{0}^{s}\left(s^{2}-r^{2}\right)^{(l-2) / 2} r^{n-1} \mathrm{~d} r .
$$

By a change of variable, $d^{\prime}=1$, concluding the proof.

Note that this result is valid for a larger range of parameters than Theorem 2.2. In particular, for $n=1$ and $l=m-1$, the result provides a geometric interpretation of the Poisson integral representation for Bessel functions.

\subsection{Relation with the Radon/Abel transforms}

The Radon transform integrates functions defined on $\mathbb{R}^{n}$ over all hyperplanes; restricted to radial functions, it is often called the Abel transform (which we denote by $\mathcal{A}_{n}$ ): an operator which acts on functions defined on $[0, \infty)$. For our purposes here, we also index the transplantation operator with the dimension, i.e. $T_{l}=T_{l, n}$. Restricting the operators to radial functions, it is easy to see that $\widetilde{T_{n-1, n} f}=\mathcal{A}_{n} \tilde{f}$. In particular, $T_{1,2}$ and $T_{2,3}$ are invertible on suitable classes of radial functions. Furthermore, $T_{1, n-1} T_{1, n}=T_{2, n}$. Hence, from (2.8), one can write an inversion formula for $T_{1, n}$.

It should be noted that our dual transform $T_{l}^{*}$ is not the same as the dual Radon transform in any case. Our dual was constructed to take advantage of the Hadamard map and form a dual to $T_{l}$ on spaces of radial functions. 


\subsection{Analytic perspectives}

As integral formulae for Bessel functions, (2.7) and (2.11) can be written, respectively, as

$$
\begin{gathered}
(\lambda r)^{-\mu} J_{\mu}(\lambda r)=\frac{1}{2^{\alpha-1} \Gamma(\alpha)} \int_{r}^{\infty}(\lambda s)^{-(\mu+\alpha)} J_{\mu+\alpha}(\lambda s)\left(s^{2}-r^{2}\right)^{\alpha-1} s \mathrm{~d} s \\
(\lambda s)^{-(\mu+\alpha)} J_{\mu+\alpha}(\lambda s)=\frac{1}{2^{\alpha-1} \Gamma(\alpha) s^{2(\mu+\alpha)}} \int_{0}^{s}(\lambda r)^{-\mu} J_{\mu}(\lambda r)\left(s^{2}-r^{2}\right)^{\alpha-1} r^{2 \mu+1} \mathrm{~d} r,
\end{gathered}
$$

where $\mu=\frac{1}{2}(n-1)$ and $\alpha=\frac{1}{2} l$. The former is a limiting form of Sonine's second integral and the latter is a variant on Sonine's first integral $[\mathbf{1 2}$, p. 45]. The second formula can be rewritten as

$$
J_{\mu+\alpha}(s)=\frac{s^{\alpha}}{2^{\alpha-1} \Gamma(\alpha)} \int_{0}^{1} J_{\mu}(s t)\left(1-t^{2}\right)^{\alpha-1} t^{\mu+1} \mathrm{~d} t,
$$

where we have set $\lambda=1$. In this form, the formula is useful in the study of Bochner-Riesz summability [10, p. 170]. Hence, the above theorems provide a geometric interpretation of integral representations of Bessel functions useful in potential problems and harmonic analysis.

It is possible to write analytic versions of the operator $T_{l}$ and its dual and modify the above techniques (intertwining properties and elliptic regularity arguments) to obtain the above limiting cases of Sonine's integrals for appropriate ranges of the parameters. However, we do not need such results in our applications below.

\section{Applications}

A simple illustration of the utility of the transplantation formulae is the extension of the reasoning underlying the Kirchhoff-Poisson formula (1.14) to even dimensions. The solution of the initial-value problem (1.1) in the case $n=2 m$ has the Fourier integral representation

$$
u(x, t)=\frac{\omega_{2 m-1}}{(2 \pi)^{2 m}} \int_{0}^{\infty} \mathcal{F}_{2 m}\left[\bar{f}_{(x)}\right](\lambda) \sin \lambda t \lambda^{2 m-2} \mathrm{~d} \lambda,
$$

where we assume that $f$ is smooth with compact support. What is needed is a formula lifting the above Fourier transform, $\mathcal{F}_{2 m}\left[\bar{f}_{(x)}\right](\lambda)$, to one on $\mathbb{R}^{2 m+1}$. If $g$ is a radial Lebesgue integrable function with compact support, then formally using Theorem 2.2 and duality we get

$$
\mathcal{F}_{2 m} g(\lambda)=\frac{\Gamma(m)}{2 \sqrt{\pi} \Gamma\left(m+\frac{1}{2}\right)} \lambda \mathcal{F}_{2 m+1}\left[T_{1}^{*} g\right](\lambda) .
$$

In order to justify this, the following lemma provides integrability properties of $T_{1}^{*} g$, a result which also finds use in the next subsection.

\section{Lemma 3.1.}

(i) Let $f \in L_{\mathrm{c}}^{1}\left(\mathbb{R}^{n}\right)$ be radial. Then $T_{1}^{*} f=g_{1}+g_{2}$, where $g_{1} \in L_{\mathrm{c}}^{1}\left(\mathbb{R}^{n+1}\right)$ and $g_{2} \in$ $L^{p}\left(\mathbb{R}^{n+1}\right)$ for any $p>1+(1 / n)$. 
(ii) Let $f$ be a radial measurable bounded compactly supported function, then $T_{1}^{*} f \in$ $L^{p}\left(\mathbb{R}^{n+1}\right)$ for any $p>1+(1 / n)$.

Proof. For (i), take $R>0$ such that $f(x)=0$ for $|x|>R$ and let $R^{\prime}>R$. Let $g_{1}=T_{1}^{*} f$ for $|x| \leqslant R^{\prime}$ and let it be zero elsewhere, and set $g_{2}=T_{1}^{*} f-g_{1}$. Let $c_{n}=$ $2 B\left(\frac{1}{2}, \frac{1}{2} n\right)^{-1}$. Then, from $(2.12)$,

$$
\begin{aligned}
\int_{0}^{R^{\prime}}\left|\tilde{g}_{1}(s)\right| s^{n} \mathrm{~d} s & \leqslant c_{n} \int_{0}^{R^{\prime}} s \int_{0}^{s}|\tilde{f}(r)|\left(s^{2}-r^{2}\right)^{-1 / 2} r^{n-1} \mathrm{~d} r \mathrm{~d} s \\
& =c_{n} \int_{0}^{R^{\prime}}|\tilde{f}(r)| r^{n-1} \int_{r}^{R^{\prime}}\left(s^{2}-r^{2}\right)^{-1 / 2} s \mathrm{~d} s \mathrm{~d} r \\
& \leqslant \text { const. } \times\|f\|_{1} .
\end{aligned}
$$

Again using (2.12), for $s>R^{\prime}$ we have

$$
\begin{aligned}
\left|\tilde{g}_{2}(s)\right| & \leqslant \frac{c_{n}}{s^{n-1}} \int_{0}^{R}|\tilde{f}(r)|\left(s^{2}-r^{2}\right)^{-1 / 2} r^{n-1} \mathrm{~d} r \\
& \leqslant \frac{c_{n}}{s^{n-1}\left(s^{2}-R^{2}\right)^{1 / 2}} \int_{0}^{R}|\tilde{f}(r)| r^{n-1} \mathrm{~d} r .
\end{aligned}
$$

It follows that

$$
\left|\tilde{g}_{2}(s)\right|^{p} s^{n} \leqslant \text { const. } \times \frac{1}{s^{n(p-1)}}
$$

and $g_{2} \in L^{p}\left(\mathbb{R}^{n+1}\right)$ if $p>1+(1 / n)$, as desired. The proof of (ii) is similar: we need only show that $T_{1}^{*} f(x)$ is bounded for $|x| \leqslant R^{\prime}$.

If $m \geqslant 2$, the integral defining the Fourier transform on the right-hand side of (3.1) is absolutely convergent. Indeed, standard estimates of Bessel functions imply that $\phi_{\lambda}^{n+1} \in$ $L^{q}\left(\mathbb{R}^{n+1}\right)$ provided that $q>2+(2 / n)$. The Hölder conjugate index would then satisfy $p<2(n+1) /(n+2)$. From the preceding lemma, $T_{1}^{*} g=g_{1}+g_{2}$, where $g_{1} \in L_{\mathrm{c}}^{1}\left(\mathbb{R}^{n+1}\right)$ and $g_{2} \in L^{p}\left(\mathbb{R}^{n+1}\right)$ for any $p>1+(1 / n)$. When $n>2$, we may choose $p$ to satisfy both inequalities, verifying that $\mathcal{F}_{2 m+1}\left[T_{1}^{*} g\right]$ is defined by an absolutely convergent integral. In the case where $n=2, T_{1}^{*} g$ is locally integrable on $\mathbb{R}^{3}$ and defines a tempered distribution. Hence, in two dimensions (3.1) should be interpreted in the sense of distributions.

Substituting into the formula for $u$ and simplifying the constants, we obtain

$$
u\left(x^{\prime}, t\right)=\frac{\omega_{2 m}}{(2 \pi)^{2 m+1}} \int_{0}^{\infty} \mathcal{F}_{2 m+1}\left[T_{1}^{*} \bar{f}_{\left(x^{\prime}\right)}\right](\lambda) \sin \lambda t \lambda^{2 m-1} \mathrm{~d} \lambda,
$$

where we are using the primed variables for $\mathbb{R}^{2 m}$. This should be compared with (1.11); we are left with understanding $T_{1}^{*} \bar{f}_{\left(x^{\prime}\right)}$. As in the previous section, we write $x=\left(x^{\prime}, x_{2 m+1}\right) \in$ $\mathbb{R}^{2 m+1}$. It is then easy to see that

$$
T_{1}^{*} \bar{f}_{\left(x^{\prime}\right)}\left(y^{\prime}\right)=\overline{(H f)}_{(x)}(y),
$$


where the overline on the right-hand side represents the spherical mean on $\mathbb{R}^{2 m+1}$. Recalling the Kirchhoff-Poisson formula (1.14), the solution to the initial-value problem (1.1) for $n=2 m$ takes the form

$$
u\left(x^{\prime}, t\right)=\frac{2^{m-1}(m-1) !}{(2 m-1) !}\left(t^{-1} \partial_{t}\right)^{m-1}\left[t^{2 m-1} \overline{(H f)}_{(x)}(t)\right] .
$$

This is equivalent to the classical form (see [3]). The point of the above rather formal computation is to indicate a direct role of Hadamard's method of descent in Fourier transform formulae.

Two other applications of the transplantation formulae are given in the following subsections.

\subsection{Fourier inversion at a point}

Herein we present an alternative development of results in [9] in the Euclidean space setting. The approach lies in applying the transplantation formulae to obtain a formula relating the Fourier transform of radial functions on $\mathbb{R}^{2 m+1}$ to that of the transplanted function on $\mathbb{R}^{2 m-1}$, i.e.

$$
\mathcal{F}_{2 m+1} f(\lambda)=\mathcal{F}_{2 m-1}\left[T_{2} f\right](\lambda)
$$

and formula (3.1) representing Hadamard's method of descent lifting computation of Fourier transforms on $\mathbb{R}^{2 m}$ to a Fourier transform on $\mathbb{R}^{2 m+1}$. Formula (3.2) is immediate if $f$ is a radial Lebesgue integrable function on the respective Euclidean space. Note that, when $m=1,(3.2)$ is the well-known relation between the Fourier transform on $\mathbb{R}^{3}$ and the Abel transform. We will apply these formulae to obtain a new proof and the extension of a result in $[\mathbf{9}]$ on Fourier inversion at a point.

Odd dimensions are treated first. Suppose that $f \in C_{\mathrm{c}}^{\infty}\left(\mathbb{R}^{2 m+1}\right)$ and is radial. The partial sum of the Fourier inversion integral at the origin is given by

$$
S_{R} f(\mathrm{O})=\frac{1}{(2 \pi)^{2 m+1}} \int_{|\xi| \leqslant R} \mathcal{F}_{2 m+1} f(\xi) \mathrm{d} \xi=\frac{\omega_{2 m}}{(2 \pi)^{2 m+1}} \int_{0}^{R} \mathcal{F}_{2 m+1} f(\lambda) \lambda^{2 m} \mathrm{~d} \lambda .
$$

Applying (3.2) iteratively, we obtain

$$
\begin{aligned}
S_{R} f(\mathrm{O}) & =\frac{\omega_{2 m}}{(2 \pi)^{2 m+1}} \int_{0}^{R} \mathcal{F}_{2 m-1}\left[T_{2} f\right](\lambda) \lambda^{2 m} \mathrm{~d} \lambda \\
& =\frac{-\omega_{2 m}}{(2 \pi)^{2 m+1}} \int_{0}^{R} \mathcal{F}_{2 m-1}\left[\Delta_{2 m-1} T_{2} f\right](\lambda) \lambda^{2 m-2} \mathrm{~d} \lambda \\
& =\frac{(-1)^{m} \omega_{2 m}}{2(2 \pi)^{2 m+1}} \int_{-R}^{R} \mathcal{F}_{1}\left[\Delta_{1} T_{2} \Delta_{3} T_{2} \cdots \Delta_{2 m-1} T_{2} f\right](\lambda) \mathrm{d} \lambda .
\end{aligned}
$$

Since

$$
T_{2} f(x)=2 \pi \int_{r}^{\infty} \tilde{f}(s) s \mathrm{~d} s, \quad r=|x|,
$$

it follows that

$$
\Delta_{2 l-1} T_{2} f=-2 \pi\left(r \tilde{f}^{\prime}(r)+(2 l-1) \tilde{f}(r)\right) .
$$


Hence, the bracketed term in the formula for the partial sum has the structural form

$\Delta_{1} T_{2} \Delta_{3} T_{2} \cdots \Delta_{2 m-1} T_{2} f(r)=(-1)^{m}(2 \pi)^{m}\left[\sum_{l=1}^{m} \gamma_{m, l} l^{l} \tilde{f}^{(l-1)}(r)+1 \cdot 3 \cdots(2 m-1) \tilde{f}(r)\right]$,

where the $\gamma_{n, m}$ are constants. Substituting into the formula for the partial sum, interchanging orders of integration and simplifying the constants, we obtain

$$
S_{R} f(\mathrm{O})=\sum_{l=1}^{m} \gamma_{m, l}^{\prime} \mathcal{F}_{1}\left[h_{l}\right](R)+\frac{1}{\pi} \int_{-\infty}^{\infty} \tilde{f}(r) \frac{\sin R r}{r} \mathrm{~d} r
$$

for suitable constants $\gamma_{m, l}^{\prime}$ and we have set $h_{l}(r)=r^{l-1} \tilde{f}^{(l)}(r)$. The integral on the righthand side is well known in the classical theory of Fourier integrals and series (see, for example, [13]); it converges to $\tilde{f}(0)$ as $R \rightarrow \infty$ under our hypothesis. The sum on the right-hand side tends to 0 since $h_{l} \in L^{1}(\mathbb{R})$ for all $l$. Hence, for nice functions and in odd dimensions, Fourier inversion can be reduced to the one-dimensional case. (Extension to other points $x \in \mathbb{R}^{2 m+1}$ and non-radial functions follows from the discussion below.)

What is needed now is an extension of (3.3) to more general classes of functions. For this, we introduce a variant $[\mathbf{9}]$ of the classical Dini condition. A function $g:[0, \infty) \rightarrow \mathbb{R}$ is said to satisfy Dini's condition at 0 if, for some $\delta>0$,

$$
\int_{0}^{\delta} \frac{|g(r)-g(0)|}{r} \mathrm{~d} r<\infty .
$$

If a radial $f \in L^{p}\left(\mathbb{R}^{2 m+1}\right)$ is such that $\tilde{f}$ satisfies Dini's condition at 0 , it follows that the integral on the right-hand side of (3.3) converges to $\tilde{f}(0)$. (The proof is classical [13].) Moreover, under this hypothesis, simple estimates show that $\tilde{f} \in L^{1}(\mathbb{R})$ (note that $\tilde{f}$ is an even function)* and hence it defines a tempered distribution on $\mathbb{R}$. In this manner, (3.3) is extended and we obtain the following result.

Proposition 3.2. Let $f$ be a radial function in $L^{p}\left(\mathbb{R}^{2 m+1}\right)$ for some $1 \leqslant p \leqslant 2$ such that $\tilde{f}$ satisfies Dini's condition at 0 . Then $\tilde{f}$ is a tempered distribution on $\mathbb{R}$, (3.3) holds and

$$
\lim _{R \rightarrow \infty} S_{R} f(\mathrm{O})=\tilde{f}(0) \Longleftrightarrow \lim _{R \rightarrow \infty} \sum_{l=1}^{m} \gamma_{m, l}^{\prime} \mathcal{F}_{1}\left[h_{l}\right](R)=0,
$$

where $h_{l}(r)=r^{l-1}(\mathrm{~d} / \mathrm{d} r)^{l} \tilde{f}(r)$.

In order to extend the previous proposition to even dimensions, we use formula (3.1). Lemma 3.1 provides a hypothesis suitable for examining good examples. A second technical lemma is needed, as follows (the proof is based on (2.12) and is left to the reader).

Lemma 3.3. Let $f$ be a radial locally integrable function on $\mathbb{R}^{n}$ with $\tilde{f}$ satisfying Dini's condition at 0 . Then $\widetilde{T_{1}^{*} f}$ satisfies Dini's condition at 0 with $\widetilde{T_{1}^{*} f}(0)=\tilde{f}(0)$.

* More generally, one can show that if $f \in L^{p}\left(\mathbb{R}^{n}\right)$ for some $1 \leqslant p<n$ with $\tilde{f}$ satisfying Dini's condition at 0 , then $\tilde{f} \in L^{1}(\mathbb{R})$. 
With the technicalities in order, we are now able to use formula (3.1) as mentioned above, in combination with Proposition 3.2, giving the following result.

Proposition 3.4. Let $f$ satisfy the hypothesis of Lemma 3.1 with $n=2 m$ with $\tilde{f}$ satisfying Dini's condition at 0 . Then

$$
\lim _{R \rightarrow \infty} S_{R} f(\mathrm{O})=\tilde{f}(0) \Longleftrightarrow \lim _{R \rightarrow \infty} \sum_{l=1}^{m} \gamma_{m, l}^{\prime} \mathcal{F}_{1}\left[h_{l}\right](R)=0,
$$

where

$$
h_{l}(r)=r^{l-1}\left(\frac{\mathrm{d}}{\mathrm{d} r}\right)^{l} \widetilde{T_{1}^{*} f}(r) .
$$

Example 3.5. We explore Fourier inversion at the origin for the characteristic function of the unit ball in two and three dimensions. When $n=3,(3.3)$ has the form

$$
S_{R} f(\mathrm{O})=\frac{1}{\pi} \int_{-\infty}^{\infty} \tilde{f}^{\prime}(r) \sin R r \mathrm{~d} r+\frac{1}{\pi} \int_{-\infty}^{\infty} \tilde{f}(r) \frac{\sin R r}{r} \mathrm{~d} r .
$$

In particular, for the characteristic function, $\tilde{f}^{\prime}(r)=\delta(r+1)-\delta(r-1)$, where $\delta$ is the Dirac delta, and hence the partial sum becomes

$$
S_{R} f(\mathrm{O})=\frac{2}{\pi} \sin R+\frac{1}{\pi} \int_{-1}^{1} \frac{\sin R r}{r} \mathrm{~d} r
$$

indicating oscillatory divergence as $R \rightarrow \infty$. In two dimensions, the partial sum operator can be written

$$
S_{R} f(\mathrm{O})=\frac{-\mathrm{i}}{\pi} \mathcal{F}_{1}\left[\widetilde{T_{1}^{*} f}\right](R)+\frac{1}{\pi} \int_{-\infty}^{\infty} \widetilde{T_{1}^{*} f}(r) \frac{\sin R r}{r} \mathrm{~d} r
$$

In particular, if $f$ is the characteristic function of the unit ball, then

$$
\widetilde{T_{1}^{*} f}(s)= \begin{cases}1, & \text { if }|s|<1, \\ 1-\left(1-\left(1 / s^{2}\right)\right)^{1 / 2}, & \text { if }|s|>1,\end{cases}
$$

and hence $T_{1}^{*} f \in L^{p}\left(\mathbb{R}^{3}\right)$ for $p>\frac{3}{2}$. Also note that

$$
{\widetilde{T_{1}^{*}}}^{\prime}(s)= \begin{cases}0, & \text { if }|s|<1, \\ \frac{1}{s^{3}\left(1-\left(1 / s^{2}\right)\right)^{1 / 2}}, & \text { if }|s|>1\end{cases}
$$

which defines an $L^{1}(\mathbb{R})$-function. Returning to the formula for the partial sum, the first term on the right-hand side tends to zero by the Riemann-Lebesgue lemma and it is easily seen that the second term tends to 1, as expected. Fourier inversion for the characteristic function was a motivating example in [8]; other interesting examples can be found in [9]. 
Fourier inversion at a point $x \in \mathbb{R}^{n}$ and for non-radial functions $f$ can be deduced from the above results by returning to (1.7) and expressing the Fourier inversion formula in the form

$$
f(x)=\frac{\omega_{n-1}}{(2 \pi)^{n}} \int_{0}^{\infty} \mathcal{F}_{n}\left[\overline{f_{(x)}}\right](\lambda) \lambda^{n-1} \mathrm{~d} \lambda .
$$

In this form, Fourier inversion at $x$ is equivalent to Fourier inversion of the Fourier transform of the spherical mean of $f$ at the origin. For notational convenience, we set $F(y)=\overline{f_{(x)}}(y)$, the spherical mean of $f$ centred at $x$ as a function on $\mathbb{R}^{n}$, and make the following definition.

Definition 3.6. A measurable function $f$ on $\mathbb{R}^{n}$ is said to satisfy Dini's condition at $x$ if, for some $\delta>0$,

$$
\int_{0}^{\delta} \frac{|\tilde{F}(r)-\tilde{F}(0)|}{r} \mathrm{~d} r<\infty .
$$

We also note that if $f \in L^{p}\left(\mathbb{R}^{n}\right)$ for some $p \geqslant 1$, then $F \in L^{p}\left(\mathbb{R}^{n}\right)$ for all $x$. The extension of the previous results to non-radial functions can now be given; the proof is immediate from our definitions and the radial case.

\section{Theorem 3.7.}

(i) Let $f \in L^{p}\left(\mathbb{R}^{2 m+1}\right)$ for some $1 \leqslant p \leqslant 2$ and let it satisfy Dini's condition at the point $x$. Then

$$
\lim _{R \rightarrow \infty} S_{R} f(x)=\tilde{F}(0) \Longleftrightarrow \lim _{R \rightarrow \infty} \sum_{l=1}^{m} \gamma_{m, l}^{\prime} \mathcal{F}_{1}\left[h_{l}\right](R)=0
$$

where

$$
h_{l}(r)=r^{l-1}\left(\frac{\mathrm{d}}{\mathrm{d} r}\right)^{l} \tilde{F}(r)
$$

(ii) Let $f \in L_{\mathrm{c}}^{1}\left(\mathbb{R}^{2 m}\right)$ and let it satisfy Dini's condition at the point $x$. Then

$$
\lim _{R \rightarrow \infty} S_{R} f(x)=\tilde{F}(0) \Longleftrightarrow \lim _{R \rightarrow \infty} \sum_{l=1}^{m} \gamma_{m, l}^{\prime} \mathcal{F}_{1}\left[h_{l}\right](R)=0
$$

where

$$
h_{l}(r)=r^{l-1}\left(\frac{\mathrm{d}}{\mathrm{d} r}\right)^{l} \tilde{F}(r)
$$

Remark 3.8. Part (i) for $p=2$ and part (ii) were obtained in [9] using an approach based on the wave equation. The underlying idea there was the connection between the focusing-of-singularities property of the wave equation and Fourier inversion. It is precisely the terms in the sum on the right-hand side of the partial sum formula (3.3) that bring about this property of the wave equation. Further results and examples can be found in [9]. Undoubtedly, the above result can be further generalized by extending part (i) to broader classes of functions and even certain classes of tempered distributions. 


\subsection{Paley-Wiener theory}

Here we provide an alternative approach to Helgason's [5] Paley-Wiener theorem for the Fourier transform on $\mathbb{R}^{n}$. A similar technique works just as well for the spectral version of this theorem found in [1]. Helgason's theorem can be stated as follows.

Theorem 3.9. Let $n \geqslant 2$. A smooth function $g(\lambda, \omega)$ on $\mathbb{R} \times S^{n-1}$ is the Fourier transform of some $f \in C_{\mathrm{c}}^{\infty}\left(\mathbb{R}^{n}\right)$ with supp $f \subset \overline{B(O, R)}$ if and only if the function $g$ has an entire extension for each $\omega \in S^{n-1}$ such that the following two conditions hold:

(i) the estimate

$$
|g(\lambda, \omega)| \leqslant C_{N}(1+|\lambda|)^{-N} \mathrm{e}^{R|\operatorname{Im} \lambda|}
$$

is valid for each $N=0,1, \ldots$;

(ii) for each spherical harmonic $Y_{k}(\omega)$ of degree $k$, the function

$$
\lambda \rightarrow \lambda^{-k} \int_{S^{n-1}} g(\lambda, \omega) Y_{k}(\omega) \mathrm{d} \omega
$$

is even and entire.

The necessity of (i) follows from standard estimates; that of (ii) follows from formulae below. Our focus here is on the sufficiency of the conditions. Define the function $f$ through the Fourier inversion formula $\left(x=r \eta, r>0, \eta \in S^{n-1}\right)$

$$
f(r \eta)=\frac{1}{(2 \pi)^{n}} \int_{0}^{\infty} \int_{S^{n-1}} g(\lambda, \omega) \mathrm{e}^{\mathrm{i} \lambda r(\eta \cdot \omega)} d \omega \lambda^{n-1} \mathrm{~d} \lambda .
$$

Once the support condition on $f$ is proved, the fact that $g=\mathcal{F} f$ follows by Fourier inversion. A key step in Helgason's proof of sufficiency is the reduction of the result to the radial case via an identity due to Bochner: let $Y_{k}$ be a spherical harmonic of degree $k$ and let $\omega$ and $\eta$ denote spherical variables in $S^{n-1}$. Then

$$
\int_{S^{n-1}} \mathrm{e}^{\mathrm{i} \lambda r(\eta \cdot \omega)} Y_{k}(\omega) \mathrm{d} \omega=\mathrm{i}^{k}(2 \pi)^{n / 2} \frac{J_{(n+2 k-2) / 2}(\lambda r)}{(\lambda r)^{(n-2) / 2}} Y_{k}(\eta) .
$$

In our notation, using (1.9) this becomes

$$
\int_{S^{n-1}} \mathrm{e}^{\mathrm{i} \lambda r(\eta \cdot \omega)} Y_{k}(\omega) \mathrm{d} \omega=\frac{\mathrm{i}^{k} \pi^{n / 2}}{2^{k-1} \Gamma\left(\frac{1}{2}(n+2 k)\right)}(\lambda r)^{k} \tilde{\phi}_{\lambda}^{n+2 k}(r) Y_{k}(\eta) .
$$

In order to see the reduction to the radial case, let $\left\{Y_{k j}\right\}$ be a complete set of orthonormal spherical harmonics. Let

$$
g(\lambda, \omega)=\sum_{k, j} g_{k j}(\lambda) Y_{k j}(\omega)
$$

be the spherical harmonic expansion of $g$. From condition (ii) the function $\lambda^{-k} g_{k j}(\lambda)$ is an even entire function and, from (i), it satisfies exponential growth conditions like those 
of $g$. Substituting this expansion into the Fourier inversion formula, interchanging the summation and integrals and applying (3.5) gives

$$
f(r \eta)=\sum_{k, j} c_{n, k} r^{k}\left[\int_{0}^{\infty} \lambda^{-k} g_{k j}(\lambda) \tilde{\phi}_{\lambda}^{n+2 k}(r) \lambda^{n+2 k-1} \mathrm{~d} \lambda\right] Y_{k, j}(\eta),
$$

where $\left\{c_{n, k}\right\}$ are constants. The support conclusion can be obtained by showing that the bracketed terms above are 0 for $r>R$. Further, the integrals in the above sum are of the form of the Fourier inversion on $\mathbb{R}^{n+2 k}$ for the radial case. This is the desired reduction to the radial case recorded in the following lemma.

Lemma 3.10. Let $n \geqslant 2$. A smooth even function $g(\lambda)$ on $\mathbb{R}$ is the Fourier transform of a radial function $f \in C_{\mathrm{c}}^{\infty}\left(\mathbb{R}^{n}\right)$ with supp $f \subset \overline{B(O, R)}$ if and only if $g$ has an entire extension satisfying the estimates

$$
|g(\lambda)| \leqslant C_{N}(1+|\lambda|)^{-N} \mathrm{e}^{R|\operatorname{Im} \lambda|},
$$

for all $N=0,1, \ldots$.

Proof. From the remarks above, all we need to demonstrate is the support conclusion for

$$
f(x)=\frac{\omega_{n-1}}{(2 \pi)^{n}} \int_{0}^{\infty} g(\lambda) \phi_{\lambda}^{n}(x) \lambda^{n-1} \mathrm{~d} \lambda .
$$

In the case where $n=2 m+1$ is odd, this can be reduced to the one-dimensional PaleyWiener theorem by using (1.13) after expressing the above equation in terms of radial variables. The even-dimensional case, $n=2 m$, is lifted to the odd-dimensional case via the transplantation formula (2.7) directly when $m \geqslant 2$. The case $n=2$ must be handled separately using Remark 2.4. (The integral (2.7) must be defined as a Cauchy limit in this case; dominated convergence applies.)

The above lemma concludes the alternative proof of Helgason's theorem.

\section{References}

1. W. O. Bray, A spectral Paley-Wiener theorem, Monatsh. Math. 116 (1993), 1-11.

2. W. O. BRay And M. A. Pinsky, Pointwise Fourier inversion on rank one symmetric spaces of non-compact type and related topics, J. Funct. Analysis 30 (1997), 306-333.

3. G. D. Folland, Introduction to partial differential equations (Princeton University Press, 1995).

4. J. HADAMARD, Lectures on Cauchy's problem in linear partial differential equations (Yale University Press, 1923).

5. S. Helgason, Groups and geometric analysis (Academic Press, 1984).

6. F. John, Plane waves and spherical means applied to partial differential equations (Springer, 1955).

7. N. N. LeBedev, Special functions and their applications (Prentice-Hall, Englewood Cliffs, NJ, 1965).

8. M. A. PINSKY, Pointwise Fourier inversion and related eigenfunction expansions, Commun. Pure Appl. Math. 47 (1994), 653-681. 
9. M. A. Pinsky AND M. TAYlor, Pointwise Fourier inversion: a wave equation approach, J. Fourier Analysis Applic. 3(6) (1997), 647-703,

10. E. M. Stein And G. Weiss, Introduction to Fourier analysis on Euclidean space (Princeton University Press, 1971).

11. R. S. Strichartz, Harmonic analysis as spectral theory of Laplacians, J. Funct. Analysis 87 (1989), 51-148.

12. S. S. Vinogradova, P. D. Smith And E. D. Vinogradova, Canonical problems in scattering and potential theory, I (Chapman \& Hall/CRC, 2001).

13. A. Zygmund, Trigonometric series (Cambridge Unversity Press, 1955). 